\title{
Are Measured Differences Between the Formants of Men, Women and Children Due to Fo Differences?
}

\author{
Maurer, Dieter ; Cook, Norman ; Landis, Theodor ; d'Heureuse, Christian
}

\begin{abstract}
Formant measurements show sex and age differences in the formant patterns of a single vowel category. Comparisons of the formant frequency values of men, women and children indicate low, middle and high values, respectively (Chiba Kajiyama 1941, Potter Steinberg 1950, Peterson Barney 1952). The differences are found for all vowel categories, and they have generally been interpreted as a consequence of different vocal tract size
\end{abstract}

DOI: https://doi.org/10.1017/s0025100300004412

Posted at the Zurich Open Repository and Archive, University of Zurich

ZORA URL: https://doi.org/10.5167/uzh-154524

Journal Article

Published Version

Originally published at:

Maurer, Dieter; Cook, Norman; Landis, Theodor; d'Heureuse, Christian (1991). Are Measured Differences Between the Formants of Men, Women and Children Due to F0 Differences? Journal of the International Phonetic Association, 21(02):66.

DOI: https://doi.org/10.1017/s0025100300004412 


\title{
Are Measured Differences Between the Formants of Men, Women and Children Due to Fo Differences?
}

\author{
DIETER MAURER, NORMAN COOK, THEODOR LANDIS, CHRISTIAN D'HEUREUSE \\ Neuropsychologische Abteilung, Neurologische Klinik, Universitätsspital Zürich, \\ Haldenbach D, CH-8091 Zürich. Switzerland
}

\section{Introduction}

Formant measurements show sex and age differences in the formant patterns of a single vowel category. Comparisons of the formant frequency values of men, women and children indicate low, middle and high values, respectively (Chiba \& Kajiyama 1941, Potter \& Steinberg 1950, Peterson \& Barney 1952). The differences are found for all vowel categories, and they have generally been interpreted as a consequence of different vocal tract size.

Early studies on vowel synthesis (Potter \& Steinberg 1950, Miller 1953) indicated, and recent investigations on synthetic vowels (Traunmüller 1981, 1985), vowel synthesizers (Bennett \& Rodet 1989) and analysis of real vocalizations (Maurer et al. 1991) have suggested a direct relationship of the formant pattern with F0. Because of this, the differences in the patterns for men, women and children could stem at least in part from the different F0 of their speech. And if so, the differences should partly disappear when F0 values of the different speaker groups are identical.

This article gives the results of a study which investigated the formant patterns of five German vowels of men, women and children both at the F0 of their normal speech and at the same F0 among the speaker groups.

\section{Methods}

In acoustic theory, formants are regarded as resonances of the vocal tract (Fant 1960), or as energy concentration positions in the speech waves, i.e., energy peaks in the spectral envelope (Joos 1948, Potter \& Steinberg 1950). Because the principal idea of our study was to reexamine the formant patterns in a manner comparable to those of Potter \& Steinberg (1950) and Peterson \& Barney (1952), we looked at the formants as energy concentration positions in the speech waves, and investigated the frequency values of the formants with LPC analysis. It is of importance to note that this investigation is made solely from the perspective of the physical properties of the vowel sound wave, and not from the perspective of speech production. The difference between these views is that a formant regarded as a maximum of the vocal tract transfer function may sometimes not be represented as an energy peak in the spectral envelope, particularly in high pitched vowels. In addition, from the viewpoints of speech production and of acoustic phonetics, there is no dependence of the formant pattern on $\mathrm{F} 0$, whereas in the analysis of real vocalizations and in vowel synthesis, such a dependency is indicated.

Our reexamination of the sex and age differences in vowel formant patterns was confined to the investigation of the means and standard deviations of the formant frequency values. Formant amplitudes and bandwidths are known to be largely irrelevant to vowel 
quality (Klatt 1982).

Two methodological problems had to be solved: The determination of formant frequencies is ambiguous for higher F0 (Peterson \& Barney 1952). LPC analysis does not always reveal the same number of formants within one vowel category, given one parameter setting for all the analyzed vowel sounds (Maurer et al. 1991). In consequence, not all vowel sounds of one category will show the formant pattern given in the literature, nor the expected formant frequency values, nor the expected number of formants. In a preliminary stage, therefore, rules for the selection of the vowel sounds to be subjected to statistical analysis were determined. Subsequently, the means and standard deviations of the formant frequencies of all the selected vowels were calculated.

\section{Data collection and selection}

Five German vowels spoken by seven men, seven women and seven children were recorded at different $\mathrm{F} 0$ to create a collection of 280 vowels with both different and equal F0's for the different speaker groups. Subsequently, the vocalizations were presented to 4 members of the Neuropsychology Unit (Zürich University Hospital) for identification. Thirdly, the formant patterns of the identified vocalizations were studied to obtain the rules of selection for statistical calculation.

Subjects: The age ranges of the men and women were from 20-40 years. The children were 9 years old. Only those men who could vocalize clearly one octave above their ordinary speech level were chosen.

Vowels and F0: We studied the German vowels [u:], [o:], [a:], [e:], and [i:] pronounced with F0 of approximately $110,170,220$, and $270 \mathrm{~Hz}$ for men, 170, 220, and $270 \mathrm{~Hz}$ for women, and $270 \mathrm{~Hz}$ for children.

Recording Procedure: For each vocalization the subject heard a standard vowel target. He or she then had to produce the same vowel on the same level of F0 and had to sustain it for at least $2 \mathrm{sec}$. The vocalization was recorded by digitizing $2 \mathrm{sec}$. of the sound at a sampling frequency of $10417 \mathrm{~Hz}$. The standard and recorded vowels were then compared, and the recording was retained if the recorded vowels were the same as the standard in terms of category and F0.

Identification Test: The recorded vocalizations were presented to four members of the Neuropsychology Unit, who gave the vowel identity in API/IPA. If the identification of at least three of them was in accordance with the recording procedure, the vowel was taken as correctly identified and was selected for statistical analysis. If not, it was excluded from further study. Eleven of the 280 vocalizations were not recognized clearly in the identification test and were excluded.

Formant Determination: In order to calculate the formant frequency values, we applied LPC analysis with the following parameter settings: Hamming window of 1048 sampling points; analyzed time frame $=0.1 \mathrm{sec}(1.0-1.1 \mathrm{sec}$ of recording); filter order $=12$; analyzed frequency range $=5 \mathrm{kHz}$; pre-emphasis $=98 \%$; formant bandwidth $<500 \mathrm{~Hz}$. 
Formant Pattern Determination: The study of the formant frequency values of the vowels confirmed the methodological problems noted above. Most importantly, LPC analysis did not reveal a constant number of formants within one vowel category. For [u:] and [o:] there were vocalizations found with only one formant frequency below $1.5 \mathrm{kHz}$. For [a:] there were vocalizations found with three formant frequencies below $1.5 \mathrm{kHz}$. On the other hand, for the vocalizations of [u:], [o:] and [a:] which showed two formant frequencies below $1.5 \mathrm{kHz}$, the third formant frequency was not found to behave as might be expected from the literature. That is, within a given speaker group, either the fourth formant frequency of a vocalization had a value equal to or below the third formant frequency of another vocalization of the same vowel category, or there was no third formant frequency found (see Jorgensen 1969, for similar results).

We therefore formulated rules for the selection of the vowels to be included in the statistical analysis: The formant pattern of a vowel had to show two formant frequencies for [u:], [o:] and [a:], and three formant frequencies for [e:] and [i:] within certain frequency regions corresponding to the formant frequencies given in the literature for German vowels (Jorgensen 1969, Wängler 1981). Table 1 shows these formant regions. For [e:] and [i:], we did not exclude F3 because of a possible interaction of F2 with F3 and because of the possible importance of this interaction for the vowel identity. Determination of F3 for these two vowels is less ambiguous than for the other vowels, but still vague for some vocalizations.

Table 1: Expected formant frequency regions in $\mathrm{Hz}$ for the five German vowels. (Values are not given separately for the different speaker groups, hence regions overlap for formant frequencies above $2 \mathrm{kHz}$.)

\begin{tabular}{|c|c|c|c|}
\hline $\begin{array}{l}\text { Vowel } \\
{[\mathrm{u}:]} \\
{[\mathrm{o}:]} \\
{[\mathrm{a}:]} \\
{[\mathrm{e}:]} \\
{[\mathrm{i}:]}\end{array}$ & $\begin{array}{c}\mathrm{F} 1 \\
\mathrm{~min} / \max \\
200-400 \\
300-600 \\
550-850 \\
300-600 \\
200-400\end{array}$ & $\begin{array}{c}2 \\
\min / \max \\
600-1000 \\
650-1200 \\
900-1600 \\
1800-2800 \\
1900-3200\end{array}$ & $\begin{array}{c}\text { F3 } \\
\min / \max \\
-- \\
-- \\
-- \\
2400-3700 \\
2500-4200\end{array}$ \\
\hline
\end{tabular}

Fourteen of the remaining 269 vocalizations showed LPC resonance patterns which did not coincide with the expected number and frequency regions of the formants as given in Table 1, and were also excluded from the analysis (Table 3, column 6 on the right). Figure 1 illustrates various cases in which LPC analysis failed to reveal the expected number of formants. Moreover, within the expected range of formant values the variations were extreme in some cases. Figure 2 shows three such examples. There were formant patterns for one vowel category for which F2 of one pattern is near to F3 of the other one, accompanied by a difference for $\mathrm{F} 3$ of the two patterns of $800 \mathrm{~Hz}$ (see Figure 2, S7-S10)! There were also patterns for which a relatively low F2 and high F3 of one vocalization represent the same vowel identity as a relatively high F2 and low F3 (see Figure 2, S11 and $\mathrm{S} 12$ ). In general, we found that particularly the formant frequencies above $2 \mathrm{kHz}$ varied strongly in a non-systematic way. 

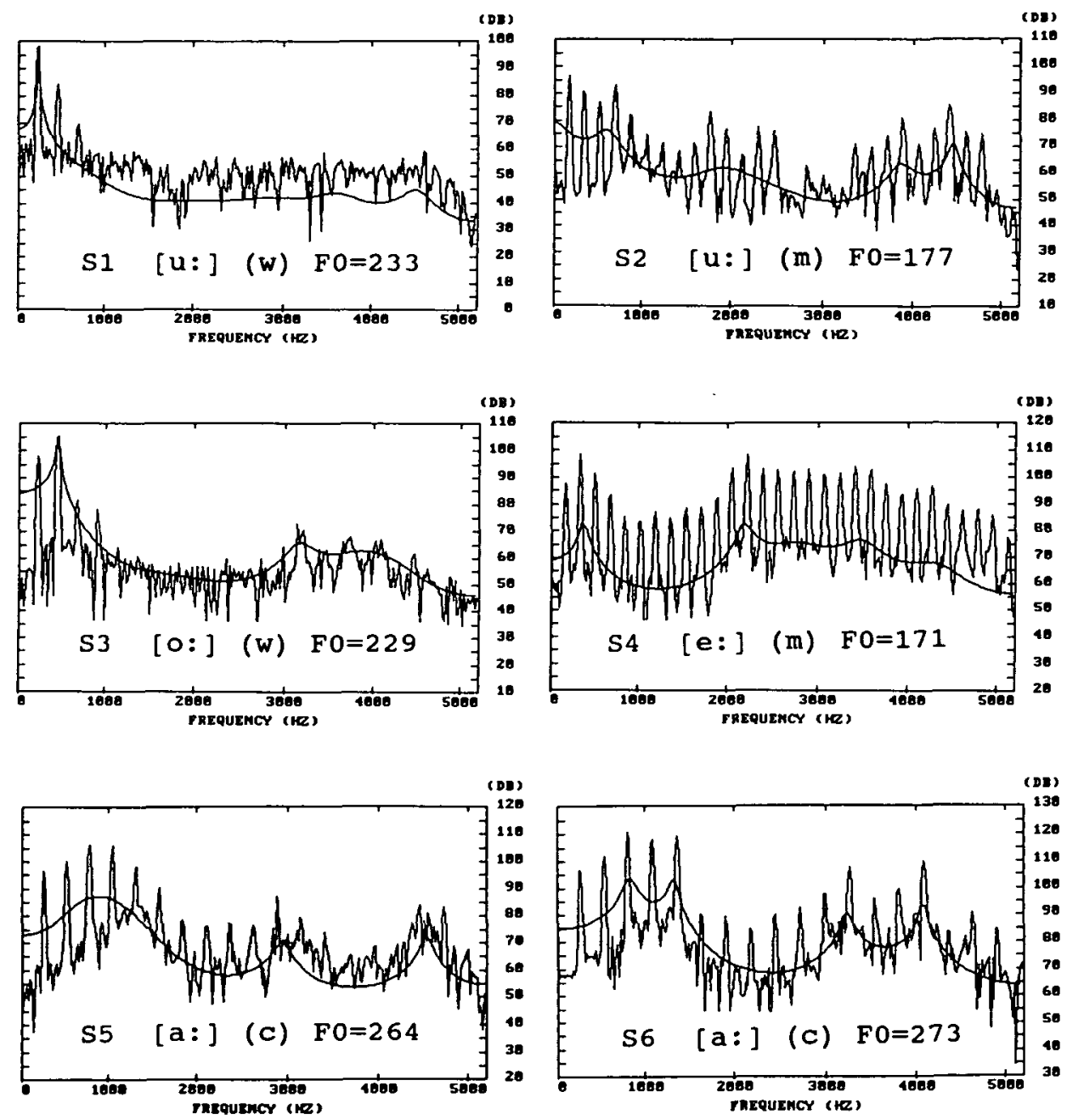

FIGURE 1: Spectra of vocalizations excluded for not coinciding with expected formant patterns in Table 1. $\mathrm{S}(\mathrm{i})=$ Spectrum number; $\mathrm{m}=$ man, $w=$ woman, $\mathrm{c}=$ child. For corresponding LPC resonance frequencies, see Table 2. S1-S3 show only one relevant formant frequency for [u:] and [o:] (S2 is a little nasal, but clearly identified). S4 represents a man's [e:] for which the determination of F3 is unclear. The LPC resonance frequency for F3 lies within the expected area of Table 1, but far exceeds the values of the other men's. For S5 there is no pattern of F1 and F2 for [a:], as found for the parallel vocalization with "normal" values in S6 (there was almost no difference in vowel color for these two [a:] ; compare also Figure 4). 

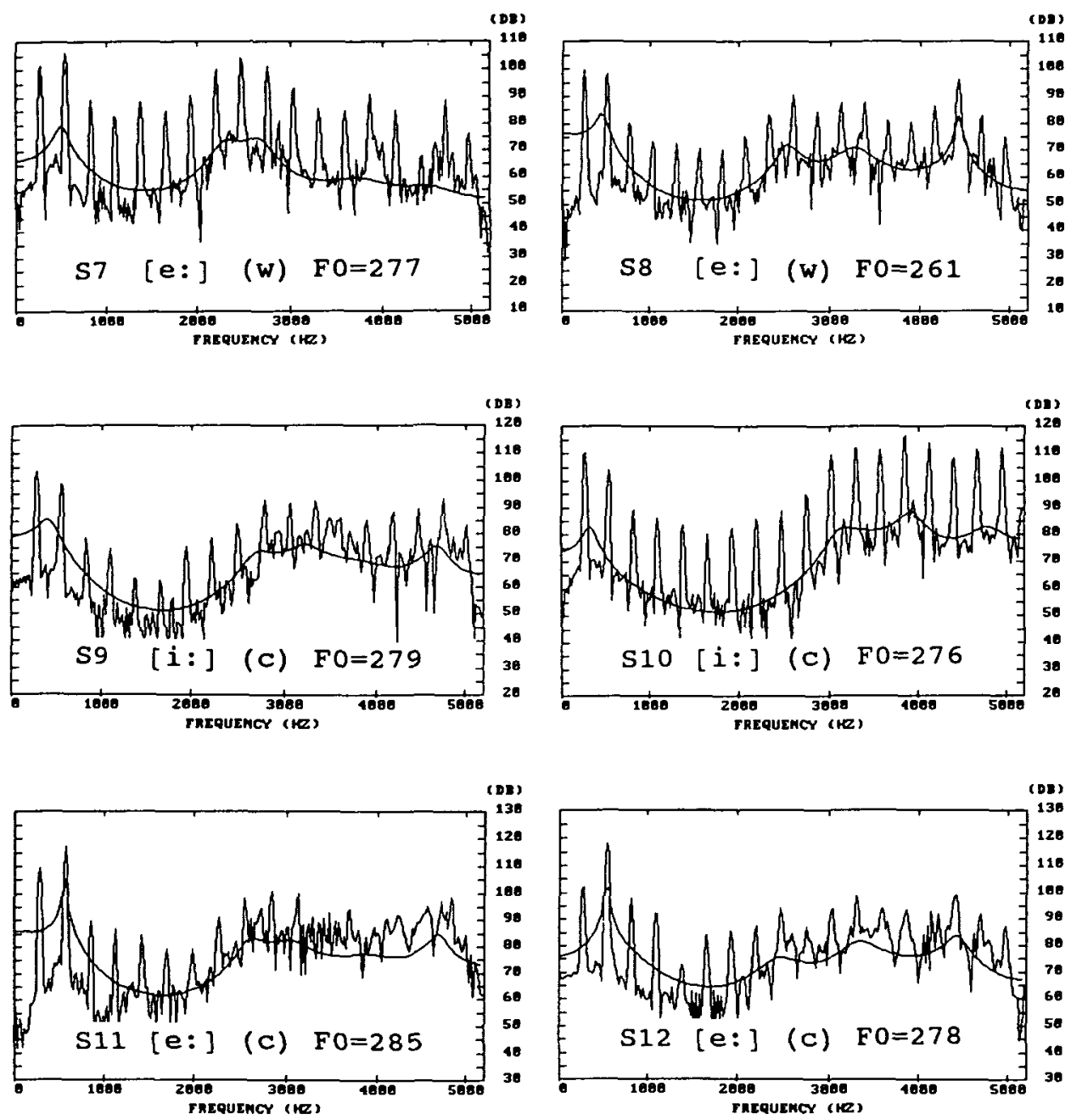

FIGURE 2: Variations of F2 and F3 for [e:] and [i:]. S7 and S8 are [e:]'s from two women. For corresponding LPC resonance frequencies, see Table 2. F3 of S7 is near to $F 2$ of S8. The variation of F3 for the two vocalizations is ca. $800 \mathrm{~Hz}$ ! S9 and S10 show the same for [i:]'s of two children. S11 and S12 show another type of variation: High F2 and low F3 for S11 in comparison with low F2 and high F3 of S12. 
Table 2: LPC resonance frequencies, bandwidths (in $\mathrm{Hz}$ ), and amplitudes (in $\mathrm{dB}$ )of the single vocalizations in Figures $1-5 . \mathrm{S}(\mathrm{i})=$ spectrum number, $\mathrm{V}=$ vowel; $\mathrm{G}=\operatorname{group}(\mathrm{m}=\operatorname{man}, \mathrm{w}=$ woman, $\mathrm{c}=$ child $) ; \mathrm{F}(\mathrm{i})=$ formant frequency; $\mathrm{B}(\mathrm{i})=$ formant bandwidth; $A(i)=$ formant amplitude.

\section{$\begin{array}{lllllllllllll}\text { S } & \text { V } & \text { G } & \text { F0 } & \text { F1 } & \text { F2 } & \text { F3 } & \text { B } 1 & \text { B 2 } & \text { B 3 } & \text { A1 } & \text { A2 } & \text { A3 }\end{array}$}

Fig. 1:

$\begin{array}{llllllrllllll}\text { S1 } & \text { [u:] } & \text { (w) } & 233 & 243 & & & 23 & & & 99 & \\ \text { S2 } & \text { [u:] } & \text { (m) } & 177 & 630 & & & 222 & & & 86 & \\ \text { S3 } & \text { [o:] } & \text { (w) } & 229 & 457 & & & 32 & & & 97 & & \\ \text { S4 } & \text { [e:] } & \text { (m) } & 171 & 378 & 2186 & 3495 & 82 & 141 & 359 & 101 & 103 & 97 \\ \text { S5 } & \text { [a:] } & \text { (c) } & 263 & 705 & 995 & 1298 & 472 & 508 & 579 & 96 & 97 & 91 \\ \text { S6 } & \text { [a:] } & \text { (c) } & 273 & 835 & 1329 & & 125 & 77 & & 113 & 113 & \end{array}$

Fig. 2:

$\begin{array}{lllllllllllrr}\text { S7 } & {[\mathrm{c}:]} & \text { (w) } & 277 & 508 & 2296 & 2681 & 120 & 253 & 245 & 99 & 94 & 95 \\ \text { S8 } & {[\mathrm{e}:]} & \text { (w) } & 261 & 467 & 2532 & 3285 & 115 & 189 & 339 & 93 & 82 & 81 \\ \text { S9 } & {[\mathrm{i}:]} & \text { (c) } & 279 & 426 & 2704 & 3230 & 183 & 265 & 359 & 96 & 83 & 86 \\ \text { S10 } & {[\mathrm{i}:]} & \text { (c) } & 276 & 337 & 3117 & 3931 & 126 & 306 & 231 & 103 & 101 & 108 \\ \text { S11 } & {[\mathrm{c}:]} & \text { (c) } & 285 & 563 & 2615 & 3076 & 27 & 253 & 399 & 115 & 93 & 92 \\ \text { S12 } & {[\mathrm{e}:]} & \text { (c) } & 278 & 551 & 2450 & 3347 & 19 & 384 & 351 & 112 & 85 & 92\end{array}$

Fig. 3:

$\begin{array}{llllllrlrr}\text { S13 } & {[\mathrm{o}:]} & \text { (m) } & 261 & 523 & 1051 & 6 & 38 & 105 & 107 \\ \text { S14 } & \text { [o:] } & \text { (w) } & 268 & 538 & 1066 & 16 & 35 & 112 & 112 \\ \text { S15 } & {[\mathrm{o}:]} & \text { (c) } & 269 & 529 & 1060 & 11 & 76 & 106 & 103 \\ \text { S16 } & {[0:]} & \text { (m) } & 238 & 479 & 895 & 19 & 66 & 115 & 104 \\ \text { S17 } & \text { [0:] } & \text { (w) } & 235 & 468 & 933 & 22 & 41 & 111 & 109 \\ \text { S18 } & \text { [0:] } & \text { (m) } & 116 & 361 & 621 & 90 & 69 & 97 & 95\end{array}$

Fig. 4:

\begin{tabular}{|c|c|c|c|c|c|c|c|c|c|c|}
\hline S19 & $(\mathrm{m})$ & 269 & 823 & 1336 & & 249 & 93 & & 105 & 111 \\
\hline 520 & $(w)$ & 263 & 789 & 1324 & & 76 & 32 & & 112 & 114 \\
\hline [a:] & (c) & 262 & 352 & 880 & 1503 & 191 & 177 & 162 & 103 & 102 \\
\hline [a:] & (m) & 240 & 814 & 1287 & & 285 & 239 & & 103 & 101 \\
\hline [a:] & (w) & 238 & 729 & 1179 & & 178 & 307 & & 109 & 108 \\
\hline [a:] & $(\mathrm{m})$ & 120 & 718 & 1176 & & 214 & 82 & & 104 & 106 \\
\hline
\end{tabular}

Fig. 5:

$\begin{array}{lllllllrllrrr}\text { S25 } & \text { [i: } & \text { (m) } & 260 & 284 & 2572 & 3067 & 57 & 50 & 121 & 102 & 103 & 101 \\ \text { S26 } & \text { [i: } & \text { (w) } & 279 & 281 & 2489 & 3296 & 5 & 174 & 215 & 95 & 96 & 103 \\ \text { S27 } & \text { [i: } & \text { (c) } & 277 & 354 & 2900 & 3163 & 135 & 550 & 512 & 96 & 91 & 95 \\ \text { S27 } & \text { [i: } & \text { (m) } & 233 & 269 & 2486 & 2988 & 70 & 203 & 312 & 106 & 101 & 101 \\ \text { S29 } & \text { [i: } & \text { (w) } & 238 & 247 & 2380 & 3323 & 28 & 131 & 140 & 107 & 90 & 101 \\ \text { S30 } & \text { [i: }] & \text { (m) } & 117 & 238 & 2219 & 3080 & 19 & 109 & 176 & 102 & 89 & 94\end{array}$




\section{Results}

The vocalizations which remained after the preliminary study were used to obtain formant statistics, and the mean and the standard deviation values of $\mathrm{F} 0$ and the formants were calculated (see Table 3).

The means and standard deviations of the formant frequencies of the analyzed vowels are shown in Table 3. These indicate four major results.

1) The formant frequencies generally rise with F0, i.e., within one speaker group the formant frequencies differ depending on the $\mathrm{F} 0$ of the vocalizations.

2) When F0 is the same there is no sex difference for F1 and F2 for [u:], [o:] and [a:], nor for F1 of [e:] and [i:].

3) The comparison of adults and children shows no or only a marginal difference for F1 and F2 for [u:], and for F1 of [o:], [a:], [e:] and [i:].

4) Above $2 \mathrm{kHz}$, all the formant frequencies of the women show higher means than the men's, and the children show the highest values.

To interpret these results, the overlapping of the formant frequency regions indicated by the standard deviations must be taken into account. To give an example of the consequence of such an overlapping, Figures 3 - 5 show comparisons of [o:], [a:] and [i:] of a man, a woman and a child. For [o:] and [a:] there is no formant pattern differences indicated below $2 \mathrm{kHz}$.

Table 3: Formant table. Means $(\bar{x})$ and standard deviations (SD) of the formant frequencies in $\mathrm{Hz}(\mathrm{m}=$ men, $w=$ women, $\mathrm{c}=$ children) grouped by $\mathrm{F} 0$ range. The numbers of excluded vocalizations based on identification (I) or spectral shape (S) are given in the last two columns.

\begin{tabular}{|c|c|c|c|c|c|c|c|c|c|c|c|}
\hline \multirow[t]{2}{*}{ Vowel } & \multirow[t]{2}{*}{ Group } & \multicolumn{2}{|c|}{ F0 } & \multicolumn{2}{|c|}{$\mathrm{F} 1$} & \multicolumn{2}{|c|}{$\mathrm{F} 2$} & \multicolumn{2}{|c|}{ F3 } & \multirow[b]{2}{*}{1} & \multirow[b]{2}{*}{$S$} \\
\hline & & $\overline{\mathrm{x}}$ & $S D$ & $\overline{\mathrm{x}}$ & SD & $\overline{\mathbf{x}}$ & $S D$ & $\overline{\mathbf{x}}$ & $S D$ & & \\
\hline \multirow[t]{8}{*}{ [u:] } & $m$ & 117 & (3) & 263 & (31) & 736 & (45) & - & - & - & - \\
\hline & $\mathrm{m}$ & 176 & (4) & 260 & (50) & 710 & (46) & - & - & - & 1 \\
\hline & $\mathbf{w}$ & 186 & (12) & 267 & (37) & 730 & (41) & - & - & 1 & 3 \\
\hline & $\mathbf{m}$ & 238 & (3) & 268 & (25) & 736 & (84) & - & . & - & - \\
\hline & $w$ & 239 & (4) & 287 & (30) & 712 & (35) & - & - & - & 2 \\
\hline & $\mathrm{m}$ & 272 & (2) & 292 & (25) & 795 & (112) & . & . & - & . \\
\hline & $w$ & 273 & (6) & 293 & (19) & 789 & $(60)$ & - & . & - & . \\
\hline & c & 275 & (5) & 308 & (27) & 727 & (64) & - & - & - & . \\
\hline
\end{tabular}




\begin{tabular}{|c|c|c|c|c|c|c|c|c|c|c|c|}
\hline \multirow[t]{2}{*}{ Vowel } & \multirow[t]{2}{*}{ Group } & \multicolumn{2}{|c|}{ F0 } & \multicolumn{2}{|c|}{ F1 } & \multicolumn{2}{|c|}{ F2 } & \multicolumn{2}{|c|}{ F3 } & \multirow[b]{2}{*}{ I } & \multirow[b]{2}{*}{$\mathbf{S}$} \\
\hline & & $\overline{\mathbf{x}}$ & $S D$ & $\overline{\mathbf{x}}$ & SD & $\overline{\mathbf{x}}$ & SD & $\overline{\mathbf{x}}$ & SD & & \\
\hline [o:] & $\mathbf{m}$ & 117 & (2) & 346 & (6) & 700 & (70) & - & - & - & - \\
\hline & $\mathbf{m}$ & 175 & (2) & 358 & (39) & 743 & (48) & - & - & - & \\
\hline & $w$ & 175 & (2) & 371 & (33) & 714 & (61) & - & - & - & - \\
\hline & $\mathbf{m}$ & 235 & (6) & 468 & (24) & 840 & (85) & - & - & - & 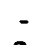 \\
\hline & $w$ & 235 & (2) & 464 & (7) & 887 & (77) & - & - & - & 2 \\
\hline & $\mathbf{m}$ & 267 & (5) & 506 & (38) & 881 & (105) & - & - & - & 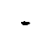 \\
\hline & $w$ & 268 & (2) & 533 & (19) & 873 & (84) & - & - & 1 & - \\
\hline & c & 274 & (7) & 547 & (11) & 1098 & (49) & - & - & - & - \\
\hline [a'] & $\mathbf{m}$ & 116 & (3) & 691 & (67) & 1099 & (82) & - & - & - & - \\
\hline & $\mathbf{m}$ & 173 & (2) & 661 & (41) & 1094 & (66) & - & - & - & . \\
\hline & $w$ & 172 & (2) & 691 & (61) & 1149 & (75) & - & - & - & - \\
\hline & $\mathbf{m}$ & 234 & (5) & 739 & (70) & 1160 & (138) & - & - & - & - \\
\hline & $w$ & 236 & (2) & 738 & (45) & 1217 & (80) & - & - & - & - \\
\hline & $\mathrm{m}$ & 264 & (7) & 746 & (65) & 1184 & (127) & - & - & . & - \\
\hline & $w$ & 263 & (2) & 757 & (85) & 1214 & (112) & - & - & - & - \\
\hline & c & 268 & (5) & 797 & (35) & 1366 & (155) & - & - & - & 3 \\
\hline [e:] & $\mathbf{m}$ & 119 & (3) & 335 & (17) & 2050 & (167) & 2633 & (132) & - & - \\
\hline & $m$ & 174 & (2) & 365 & (17) & 2098 & (181) & 2669 & (102) & - & I \\
\hline & $w$ & 177 & (4) & 373 & (24) & 2315 & (79) & 2992 & (152) & - & 1 \\
\hline & $\mathrm{m}$ & 232 & (5) & 444 & (23) & 2090 & (240) & 2752 & (133) & 1 & - \\
\hline & $w$ & 236 & (3) & 457 & (14) & 2310 & (57) & 2964 & (181) & 1 & - \\
\hline & $\mathrm{m}$ & 271 & (2) & 468 & (85) & 2120 & (138) & 2797 & (158) & 3 & - \\
\hline & $w$ & 271 & (6) & 504 & (33) & 2327 & (116) & 2889 & (251) & 1 & 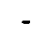 \\
\hline & $c$ & 277 & (4) & 551 & (8) & 2569 & $(100)$ & 3431 & (202) & 1 & \\
\hline [i:] & m & 120 & (3) & 247 & (18) & 2165 & (126) & 2953 & (170) & - & \\
\hline & $m$ & 177 & (2) & 285 & (39) & 2275 & (188) & 2959 & (157) & - & \\
\hline & $w$ & 178 & (2) & 297 & (51) & 2327 & (125) & 3292 & (200) & - & \\
\hline & $\mathrm{m}$ & 235 & (4) & 268 & (23) & 2292 & (247) & 2906 & (196) & - & \\
\hline & $w$ & 237 & (3) & 258 & (17) & 2435 & (113) & 3507 & (298) & - & \\
\hline & $\mathrm{m}$ & 274 & (8) & 288 & (10) & 2404 & (234) & 2941 & (136) & 1 & \\
\hline & $w$ & 277 & (2) & 287 & (9) & 2490 & (183) & 3355 & (172) & . & 1 \\
\hline & c & 277 & (4) & 325 & (35) & 2868 & $(160)$ & 3497 & (203) & 1 & \\
\hline
\end{tabular}




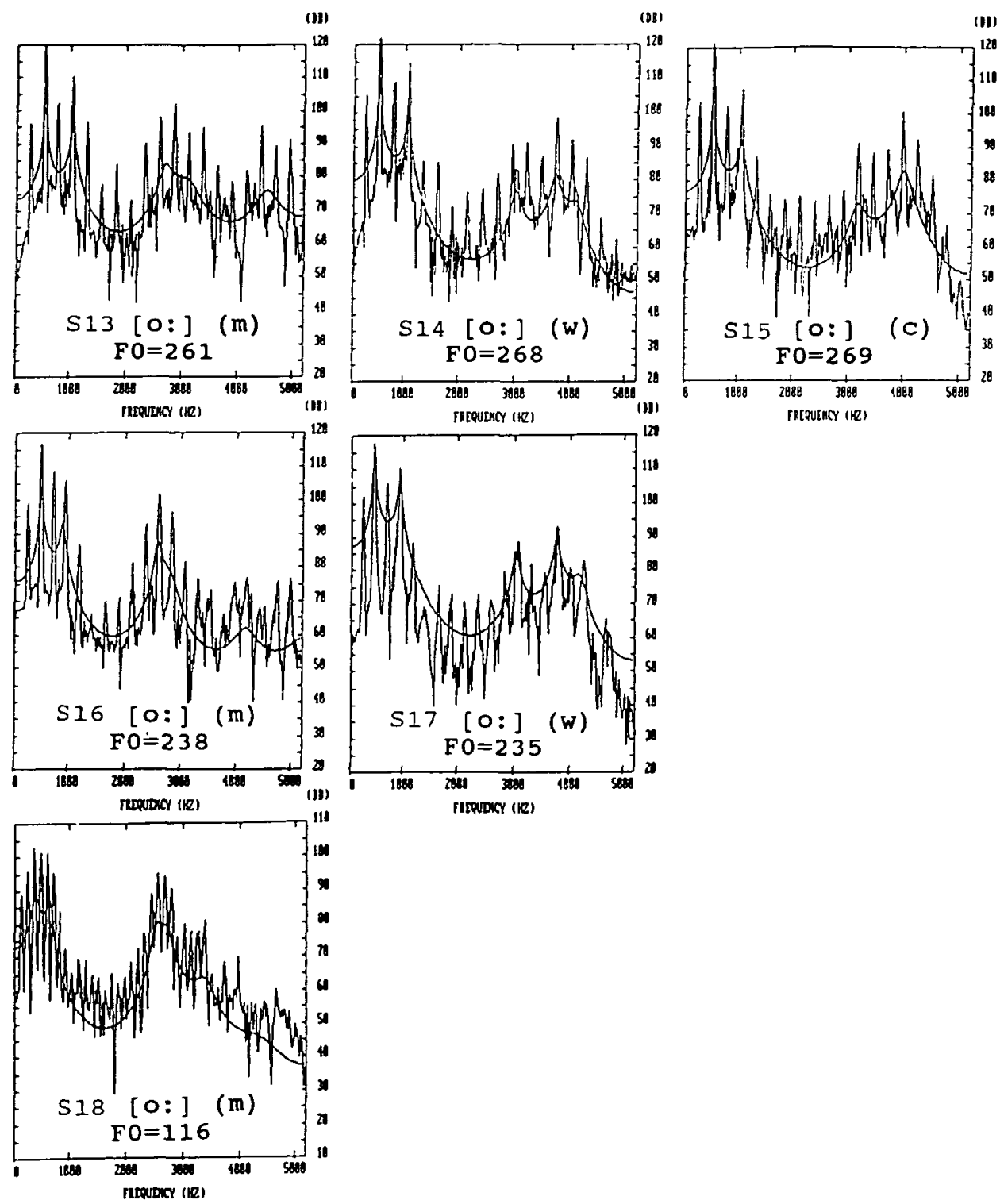

Figure 3

FIGURE 3: Vocalizations of [o:] of a man, a woman and a child. For corresponding LPC resonance frequencies, see Table 2 . No formant pattern differences were found for the same F0, but formant pattern differences within a speaker group were found for different F0. 

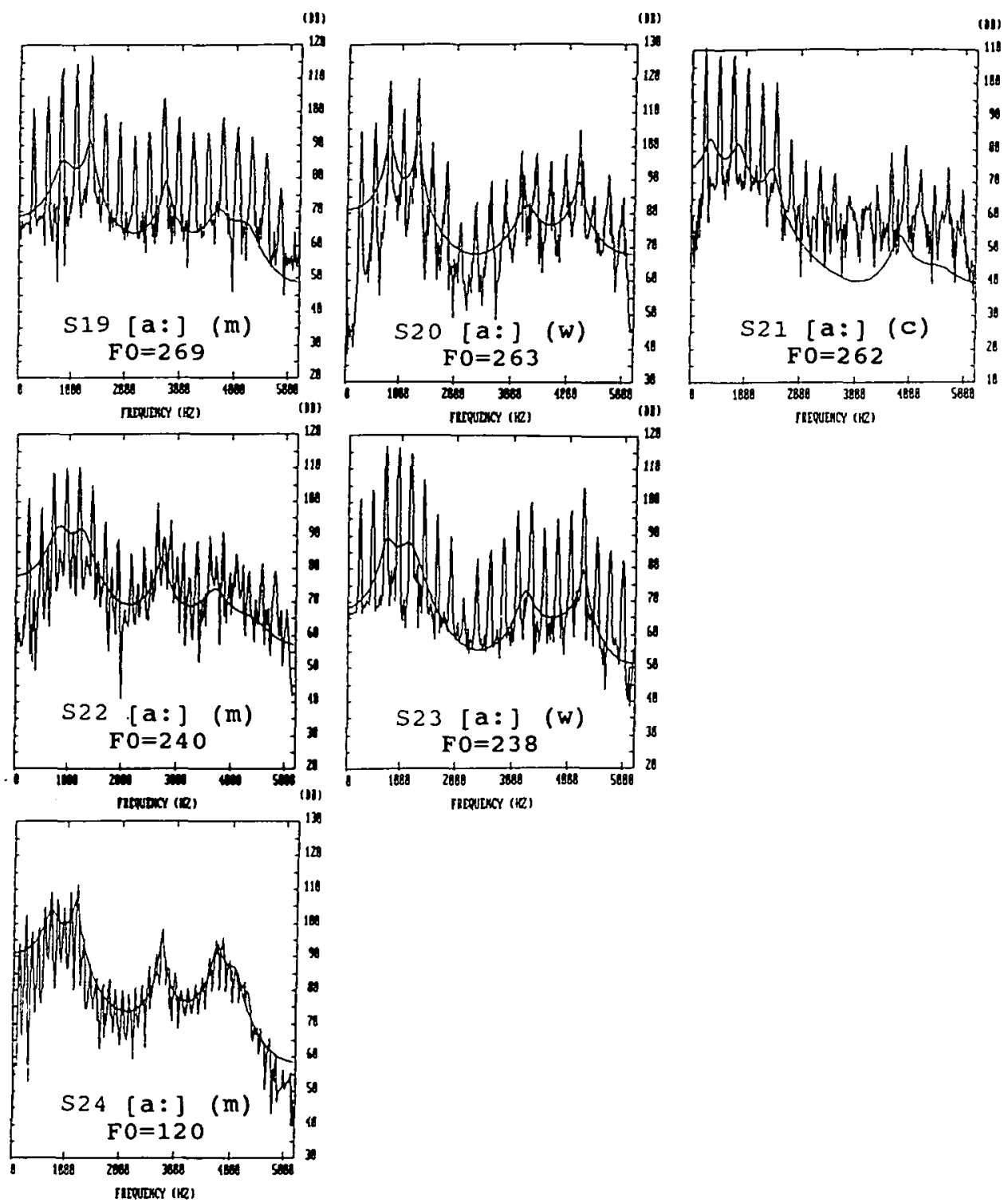

Figure 4

FIGURE 4: Vocalizations of [a:] of the same speakers as in Figure 3. For corresponding LPC resonance frequencies, see Table 2 . There were no formant patter differences for the man and woman. The formant determination of the child's vowel is unclear, but there is no indication of higher values for F1 and F2 in comparison with the man and the woman. 

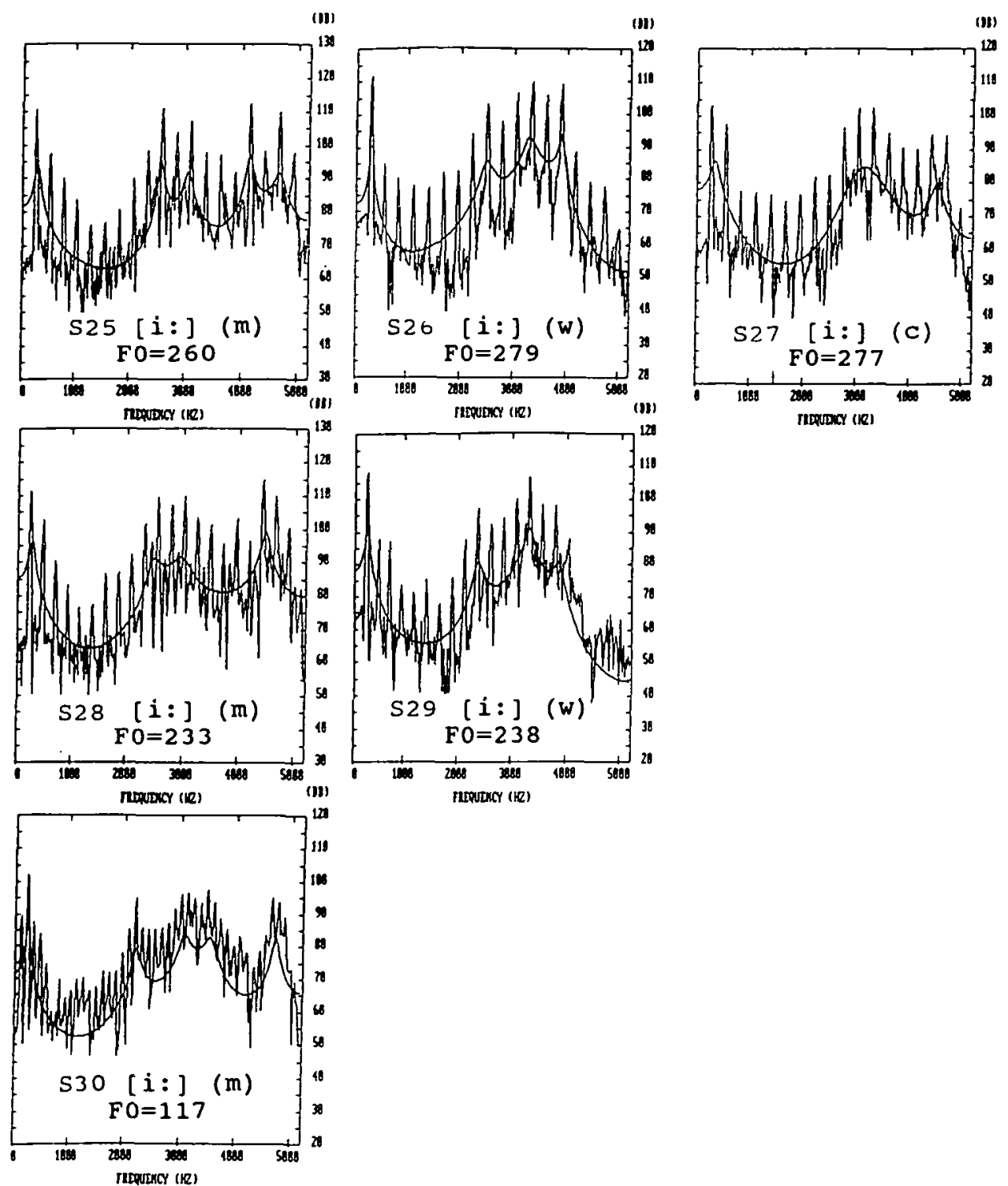

Figure 5

FIGURE 5: Vocalizations of [i:] of the same speakers as in Figure 3. For corresponding LPC resonance frequencies, see Table 2 . No formant pattern differences were found for F1 and F2 comparing the man and woman, but different F3 were found. The child shows a higher formant pattern than the adults. 


\section{Discussion}

The main purpose of this investigation was to reexamine the sex and age differences in formant patterns in order to determine whether they are due to different sex or age, or whether they are due to differences in F0. When men, women and children vocalize at the F0 of their ordinary speech, the formant patterns differ for the different speaker groups. This result corresponds to the formant statistics given in the literature, notably to the formant frequency values given by Potter \& Steinberg (1950) and Peterson \& Barney (1952). When the F0 of the different speaker groups was the same, for F1 in all vowels and for F1 and F2 in back vowels, the differences between men and women, and most of those between adults and children disappeared. That is, the lower formants shift with F0. This result confirms a dependence of the formant patterns on F0 as previously shown in studies on vowel synthesis and the analysis of real vocalizations.

In contrast to the results for the lower frequency formants, the results for those above $2 \mathrm{kHz}$ are somewhat difficult to interpret. The standard deviations of these formant frequencies are large, and an overall pattern of changes is not found. For example, some values indicate a shift of the formants with F0 (e.g., F2 of the men for [i:]) and some do not (e.g., F2 of the women for [e:]). In general, children show higher frequency values for [e:] and [i:] than women, and these two groups show higher values than men even when F0 is the same. Figure 6 illustrates these findings for the two vowels [o:] and [e:].

FIGURE 6: Mean formant frequencies $F 1$ and $F 2$ of [e:] and [o:] spoken by men $(\mathrm{m})$, women $(w)$, and children (c) at various F0's (for the corresponding frequency values see Table 3).

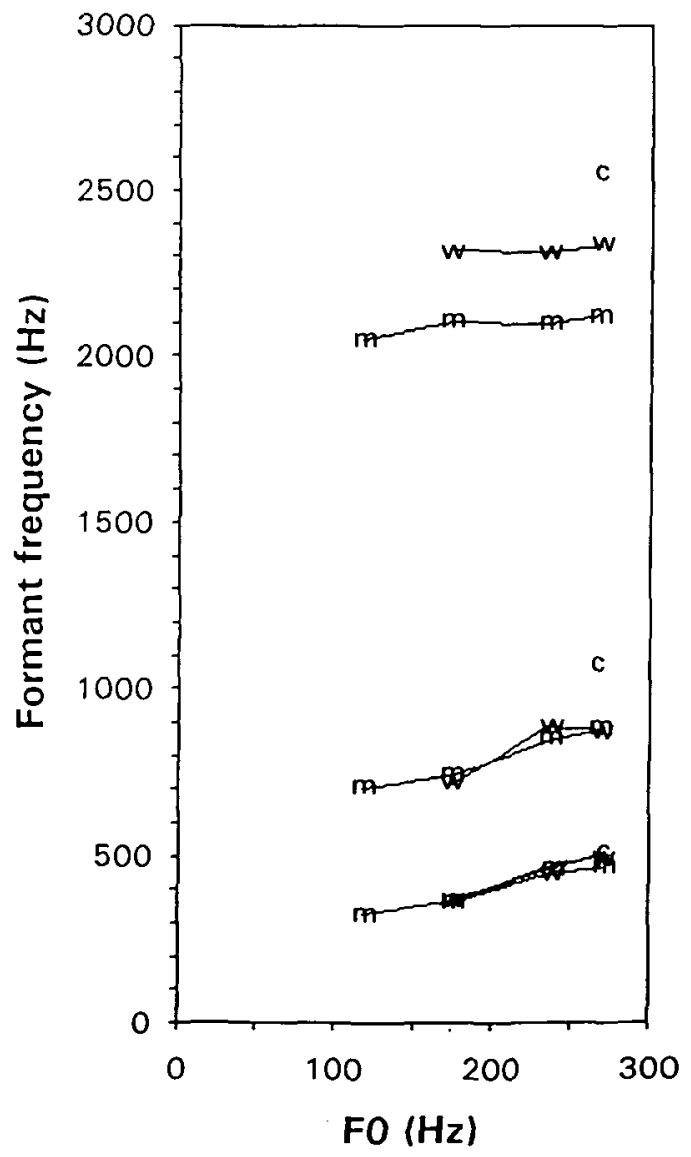

F2 [e]

F2 [o]

F1 [e], [c 
We conclude that there is no indication of general sex and age differences of the formant patterns in our results. Such differences would have to be demonstrated at equal F0 and for all the formant and for all the vowel categories, but were not found. Sex and age differences seem to be related only to higher formant frequencies.

It should be emphasized that such an interpretation is consistent with what is known from vowel synthesis. For example, if in synthesis a vowel sound is produced with a constant formant pattern, but F0 is altered, the vowel identity often changes. Conversely, if one tries to hold the identity of the synthesized vowel sound constant, the formant pattern must be changed with altering F0. What is true for synthesis is also indicated for real vocalizations. Similar phenomena should therefore be found in the analysis of real vocalizations as are found in synthesis because both are related to the perceptual identification of the sound.

The high vocalizations of the men can be considered as shouted or sung. Traunmüller (1988) showed that variations in vocal effort affect $F 0$ and the formant frequencies in the sense of a linear compression/expansion of the distances between them, if scaled logarithmically or in Bark. Given such a scaling, a linear compression is found in our results for men. This indicates that the effects reported by Traunmüller (1988) are not limited to variations in vocal effort, but can also be observed when F0 is altered for some other reason. On the other hand, studies of sung vowels show a shift of the formant frequencies with F0 without invoking the concept of vocal effort: The differences of the formant frequencies for men and women disappear for equal F0, while they appear within one singer group for different F0 (Bloothooft \& Plomp 1985). Our results are congruent with this finding.

There is an interesting difference between the productional perspective on the one hand, and the perspectives of perception and of the physical description of the vowel sound wave on the other. In production theory the formants as resonances of the vocal tract are independent of F0. Conversely, in the perspectives of perception and of the physical description of the sound wave, there is a clear indication of a direct relationship between the formant frequencies and FO. Our results confirm such a relationship. In consequence, and given a perceptual or a descriptional perspective (including vowel synthesis), it can be said that a formant pattern does not determine a vowel identity entirely because of its dependence on F0. Different formant patterns can represent one vowel category for one speaker group. (It must be emphasized that such differences in the formant patterns do not affect the vowel identity itself.) Conversely, there are examples of vocalizations within one speaker group for which a given formant pattern represents different vowel categories with different F0 (Maurer et al. 1991). Therefore, and in the light of the results of the present reexamination, we conclude that the sex and age differences of the formant frequencies below $2 \mathrm{kHz}$ found in vocalizations at the ordinary F0 of speech of the different speaker groups must be explained primarily by the difference of F0 of the vocalizations. We suggest that the articulatory process relates principally to the F0 of the vocalization. 


\section{Acknowledgement}

This study was supported by the Janggen-Pöhn Stiftung (St. Gallen), and the EMDOStiftung (Zürich).

\section{References}

BENNETT, G. AND RODET, X. (1989). Synthesis of the singing voice. In Matthews, M.V. and Pierce, R. (editors), Current Directions in Computer Music Research. Cambridge, Mass.: M.I.T. Press, 19-44.

BLADON, A. (1982). Arguments against formants in the auditory representation of speech. In Carlson, R. and Granström, B. (editors), The Representation of Speech in the Peripheral Auditory System : New York: Elsevier Biomedical Press, 95-102.

BLOOTHOOFT, G. AND PLOMP, R. (1985). Spectral analysis of sung vowels II: The effect of fundamental frequency on vowel spectra. Journal of the Acoustical Society of America 77, 1580-8.

ChiBA, T. AND KaJIYAMA, M. (1941). The Vowel: its Nature and Structure. Tokyo: Tokyo-Kaiseikan.

JORGENSEN, H.P. (1969). Die gespannten und ungespannten Vokale in der norddeutschen Hochsprache mit einer spezifischen Untersuchung der Struktur der Formantfrequenzen. Phonetica 19, 217-45.

Joos, M. (1948). Acoustic Phonetics (Language Monograph 23). Baltimore: Linguistic Society of America.

KLATT, D.H. (1982). Prediction of perceived phonetic distance from critical-band spectra: A first step. Proceedings of the International Conference on Acoustics, Speech and Signal Processing (ICASSP) 1982, 1278-81.

MAURER, D., LANDIS, T. AND D'HEuREuSE, C. (1991). Formant movement and formant number alternation with rising F0 in real vocalizations of the German vowels [u:], [o:] and [a:]. International Journal of Neuroscience 57, 25-38.

MILLER, R.L. (1953). Auditory tests with synthetic vowels. Journal of the Acoustical Society of America 25, 114-21.

PETERSON, G.E. AND BARNEY, H.L. (1952). Control methods used in a study of the vowels. Journal of the Acoustical Society of America 24, 175-84.

POTTER, R.K. AND STEINBERG, J.C. (1950). Toward the specification of speech. Journal of the Acoustical Society of America 22, 807-20.

TRAUNMÜller, H. (1981). Perceptual dimension of openness in vowels. Journal of the Acoustical Society of America 69, 1465-1475.

TRAUNMÜLLER, H. (1985). The role of the fundamental and the higher formants in the perception of speaker size, vocal effort and vowel openness. PERILUS 4, (Institute of Linguistics, University of Stockholm) 92-102.

TRAUNMÜLLER, H. (1988). Paralinguistic variation and invariance in the characteristic frequencies of vowels. Phonetica 45, 1-29.

WÄNGLER, H.H. (1981). Atlas der Deutschen Sprachlaute. Berlin: Akademie Verlag, 2022. 